\title{
Treatment time and outcome of thrombolytic therapy with strepto- kinase for acute ST Segment Elevation Myocardial Infarction (STEMI) in a District General Hospital of Sri Lanka: an audit
}

Ranasinghe $\mathbf{W} \mathbf{G}^{1}$, Kitsiri $\mathbf{P} \mathbf{H}^{\mathbf{R}^{2}}$, Sutharson $\mathbf{R}^{3}$, Dissanayaka $\mathbf{U}^{3}$ Adikari $\mathbf{C}^{3}$, Wijegunarathna $\mathbf{W}^{4}$ Sendanayaka $\mathbf{J} \mathbf{R}^{4}$, Jayamuni $\mathbf{J} \mathbf{N}^{4}$, Vithanage $\mathbf{H}^{4}$

Journal of the Ceylon College of Physicians, 2014, 45, 28-31

\begin{abstract}
An audit was carried out to determine the treatment time and outcome of a convenient sample of acute uncomplicated STEMI patients who received thrombolysis with streptokinase between August 2013 and October 2014 at the District General Hospital, Kalutara (DGHK), with a view to optimizing STEMI care in the region.

Sixty-nine patients were analyzed for Ischemic Time, Door to Needle Time and in-hospital mortality, adverse cardiac events and complications following thrombolysis.

Median Ischemic and Door to Needle Times were 3 hours and 50 minutes respectively. The proportion of patients that achieved guidelinerecommended Door to Needle Time of 30 minutes and an optimal Ischaemic Time of 2 hours was low (30\%). Although in-hospital mortality rate (3\%) is low, $35 \%$ of patients had one or more complications following thrombolysis before discharge. Hypotension was the commonest complication (17\%), while neither intracranial bleeding nor other adverse reactions to thrombolytic agent was reported.

The low proportion that received guidelinerecommended treatment times and the high proportion of in-hospital complications following thrombolysis indicate the need for optimizing STEMI care.
\end{abstract}

\section{Introduction}

Percutaneous Coronary Intervention $(\mathrm{PCl})$ centers are limited to the major cities of Sri Lanka. Centers that perform primary PCls for acute ST segment Elevation Myocardial Infarction (STEMI) in a timely

\footnotetext{
${ }^{1}$ Consultant Cardiologist

${ }^{2}$ Research Assistant, Colombo

${ }^{3}$ Consultant Physician

${ }^{4}$ Medical Officer, District General Hospital, Kalutara, Sri Lanka.
}

manner are even fewer. In most state sector hospitals reperfusion is by way of thrombolysis with streptokinase.

Additionally, national data on STEMI incidence, prevalence, treatment modes and their outcomes are scarce. The efficiency of streptokinase therapy for STEMI from an acute medical unit of Colombo South Teaching Hospital was reported in 2012. ${ }^{1}$ However, the efficiency of treatment and STEMI care from the periphery has not been reported. The absence of catheterization facilities or a rapid transfer system for primary $\mathrm{PCl}$ at District General Hospital Kalutara (DGHK) in the Western Province of Sri Lanka, leaves thrombolysis with streptokinase as the only option. Therefore we aimed to study the outcome of standard STEMI care at DGHK.

Benefits of reperfusion therapy for acute STEMI are time dependent. ${ }^{2,3}$ When streptokinase (SK) is administered as the thrombolytic agent mortality reduction is highest when treatment is started within 3 hours of onset of symptoms. ${ }^{4}$ Therefore measurement of times taken to treatment along with the outcomes are important in order to assess the quality of care provided by any STEMI care center. ${ }^{5,6}$ This data is also important in building STEMI networks and systems of care around existing STEMI centers. ${ }^{7,8}$

This audit aimed to find out the treatment times and outcomes of a convenient sample of acute uncomplicated STEMI that underwent reperfusion with thrombolytic therapy using streptokinase at DGHK in the Western Province of the country.

\section{Methodology}

Sixty-nine patients who received thrombolysis with streptokinase for acute STEMI, at DGHK in the Western Province of Sri Lanka were analyzed. We included uncomplicated acute STEMI patients presenting to DGHK between August 2013 and October 2014 (15 months) who received streptokinase as the thrombolytic therapy. We excluded patients with contraindications for streptokinase, hemodynamic instability and arrhythmia. A structured pre-tested data entry form was used for data collection. Data was 
collected by attending medical officers supervised by the treating physician. Treatment times were measured using Ischaemic Time and Door to Needle Time, as per guidelines of the European Society of Cardiology (ESC) and the American College of Cardiology Foundation/ American Heart Association (ACCFI AHA). ${ }^{5,6}$ The mean and median were used to describe the central tendencies while standard deviation and range were used to describe the spread of the treatment times. The proportions that had the Ischaemic and Door to Needle times within the guideline recommended delays and/or delays found to be optimal by currently accepted evidence were used to assess the systems efficiency in providing STEMI care. The standard delay recommended for Door to Needle Time of 30 minutes was used to assess system delays. Delays considered for Ischaemic Time were categorized as $\leq 6,3$ and 2 hours. The in-hospital outcomes were measured by overall mortality and adverse cardiac events and complications following thrombolysis before discharge. Basic demography, risk factors and duration of the hospital stay were also determined using statistical package Microsoft Excel. The audit was approved by the Institutional Head.

\section{Results}

The 69 patients included 74\% (51) males and 26\% (18) females with Male: Female ratio of approximately 3:1. Age ranged from 36 to 89 years (males 36 to 78 years and females 45 to 89 years). Mean age was $61 \pm 11$ years (males 59 versus females 65 ). Risk factor prevalence was: smoking 46\%; hypertension 45\%; dyslipidemia $39 \%$; family history of ischemic heart disease $38 \%$ and type 2 diabetes mellitus 25\%.

Ischaemic Time varied from 60 to 1115 minutes (1-18.6 hours); mean 225 minutes (3.8 hours) \pm 187 minutes (3.1 hours). Median Ischaemic Time was 180 minutes (3 hours). Approximately $90 \%$ had an Ischaemic Time of 6 hours or less (Figure 1B) while more than $50 \%$ of the sample achieved an Ischemic Time of less than 3 hours (Figure 1A). Ischemic Time of 2 hours or less was achieved only in about $30 \%$ (Figure 1).

Door to Needle Time varied from 10 to 565 Minutes (0.2-9.4 hours). Mean Door to Needle Time was 66 minutes (1.1 hours) \pm 74 minutes (1.2 hours); median 50 minutes. The proportion that accomplished the guideline recommended Door to Needle Time of less than 30 minutes, ${ }^{5,6}$ was $30 \%$ (Figure 2).

There were no complications in 36 (52\%), while 24 (35\%) had some form of complication with incomplete data in 9 (13\%). Two patients died in hospital. Both died on day three of hospital stay.
Persisting or recurring angina was experienced by $6(9 \%)$. Other complications reported included: hypotension 12 (17\%); bleeding in 4 (6\%) (hematemesis in 2, hemoptysis in 1, Gum bleeding in 1) and bradycardia in 3 (4\%) (Figure 3). There were no interhospital transfers following thrombolysis prior to discharge among the sample studied. Intracranial bleeding did not occur. Hospital stay ranged from 2 to 12 days; mean $4.7 \pm 1.3$ days.

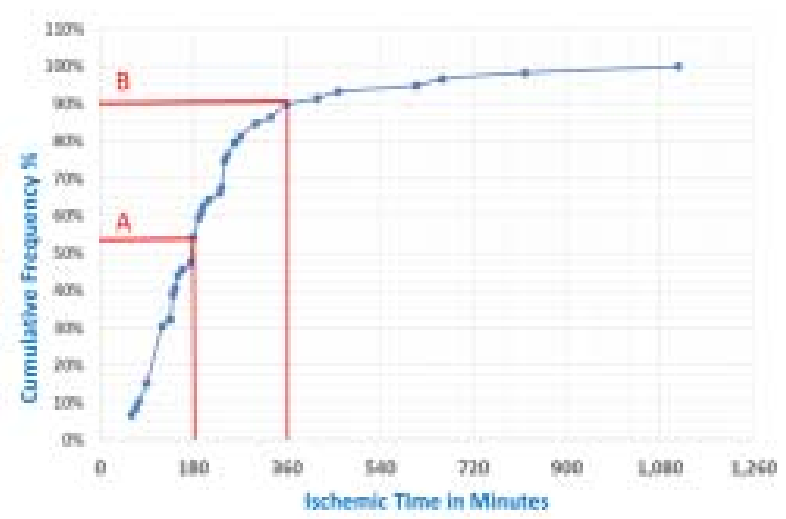

Figure 1. Cumulative Frequency of Ischaemic Time

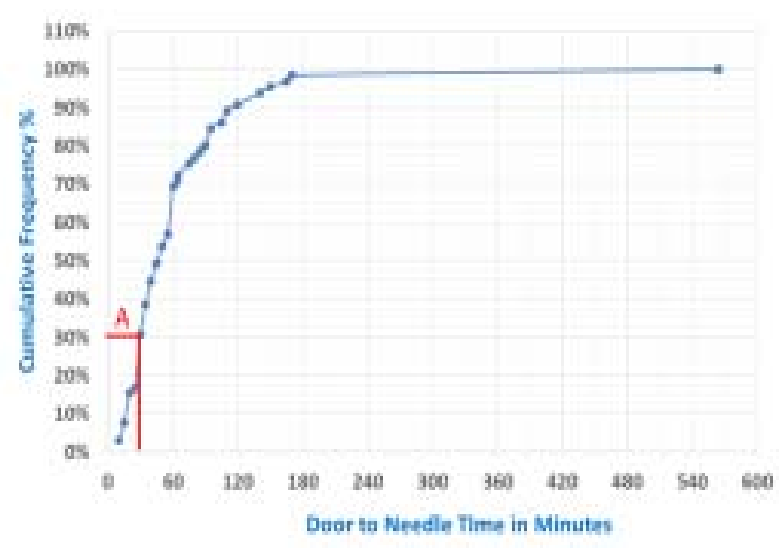

Figure 2. Cumulative Frequency of Door to Needle Time

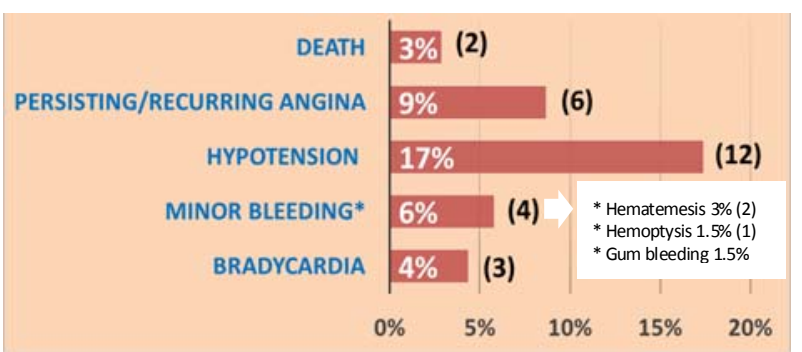

Figure 3. In-hospital outcome by complication (Categories are overlapping except with death, as some had more than one complication) 


\section{Discussion}

Data from the national registries in European countries have revealed that in-hospital mortality of unselected STEMI patients range from $6 \%$ to $14 \% .^{5}$ Therefore the $3 \%$ low mortality rate for uncomplicated STEMI found in our sample may be due to small sample size and selection bias.

It is worth noting that both in-hospital deaths occurred during day 3 of the hospital stay. Additionally $9 \%$ had persisting or recurring angina prior to discharge. Therefore implementation of a transfer protocol as per the guideline $\mathrm{s}^{5,6}$ where patients are transferred to a $\mathrm{PCl}$ capable center following thrombolysis is indicated to reduce morbidity and mortality. The recommendations are to immediately transfer those patients in whom thrombolysis has failed for Rescue PCl. ${ }^{5,6}$ In addition those who develop recurrent ischaemia or re-occlusion and/or heart failure also require immediate transfer. A pharmaco-invasive strategy is required for those transferred for early angiography and angioplasty within 3 to 24 hours of successful thrombolysis ${ }^{5,6}$ in order to further optimize care.

The previous study done in Sri Lanka in the Colombo South Teaching Hospital (CSTH) during 2008/ 2009 found that $56 \%$ of patients with STEMI responded to streptokinase when measured by $\geq 50 \%$ ST segment resolution of 12 lead ECG at 90 minutes following initiation of streptokinase. ${ }^{1}$ The response rate was highest in those who had inferior STEMI (73\%). However the response rate of anterior STEMI was still higher $(44 \%)$ than those with antero-lateral $(13 \%)$ STEMI,emphasizing the successful role of streptokinase when infarctions are confined to a smaller area of the myocardium. These findings may offer some guide to triage and transfer of STEMI patients for pharmaco-invasive strategy following thrombolytic therapy in the resource poor local peripheral settings.

Both ESC and ACCF/AHA guidelines recommend fibrin-specific agents over non fibrin-specific agents for thrombolysis. Streptokinase which is the treatment of choice in the state sector of Sri Lanka is not fibrinspecific. Furthermore, the patency rate of streptokinase at 90 minutes is lower (40\%-60\%) when compared to fibrin-specific agents (80\%). ${ }^{9}$ Although the risk of intracranial bleeding is higher with fibrin-specific agents, the mortality benefit far outweighs this risk. The most recent ESC guidelines based on evidence generated by Global Utilization of Streptokinase and Tissue plasminogen activator for Occluded coronary arteries (GUSTO) trial states that for every 1000 patients treated 10 fewer deaths occurred with fibrin specific tissue plasminogen activator (tPA) compared with streptokinase. ${ }^{5}$ There were only 3 additional strokes with tPA out of which only one caused lasting residual neurological deficit. ${ }^{5}$ In our sample the proportion who had some form of adverse effect or complication following streptokinase is relatively high (35\%) including a total of $12 \%$ deaths and recurrent or persisting angina. The CSTH study too found that 38\% of the responders and $28 \%$ of non-responders to streptokinase in their study developed complications following treatment, commonest complication being Left Ventricular Failure (16\%). ${ }^{1}$ These findings favour changing the thrombolytic agent for a guideline recommended fibrin-specific one instead of the currently used streptokinase. This is the trend not only in the developed world but also in India. ${ }^{10}$

Thrombolysis for reperfusion is recommended up to 12 hours of symptom onset. ${ }^{5,6}$ In our sample with a median Ischaemic Time 180 minutes (3 hours) about $90 \%$ had an Ischaemic Time of 6 hours or less with only 2 patients having a total Ischaemic Time greater than 12 hours. However mortality reduction is highest when reperfusion is accomplished within 3 hours of symptom onset where myocardial salvage is thought to be maximum. ${ }^{3,4}$ More than $50 \%$ of the sample achieved this Ischaemic Time of less than 3 hours. In addition the current trend is to initiate thrombolysis within the first 2 hours of symptom onset ${ }^{5}$ for the best outcome. It is not surprising that this was achieved only in about $30 \%$ of the sample, as such short Ischaemic Times mainly are a result of pre-hospital thrombolysis which is currently absent in Sri Lanka. Regional support systems for STEMI care with Emergency Medical Services (EMS) and trained paramedics for pre-hospital thrombolysis are ideal, along with the newer thrombolytic agents that could be administered as a bolus when compared to streptokinase which requires an infusion.

The key indicator of the system delay within the institution is Door to Needle Time ${ }^{5,6}$. With a median of 50 minutes, in our sample the proportion that accomplished the guideline recommended Door to Needle Time of less than 30 minutes, ${ }^{5,6}$ is limited to a mere $30 \%$, reflecting the need to implement an institutional STEMI plan to achieve shorter Door to Needle Times. The authors of the CSTH study too found the Door to Needle Times to be "alarmingly prolonged" in their setting and delay in taking the diagnostic ECG as one of the reasons. ${ }^{1}$ In this light it is also important to find out time taken for the first ECG. The guideline permitted delay is 10 minutes. ${ }^{5,6}$ Future studies should aim to measure this delay and other possible reasons that increase the Door to Needle Time. Creating awareness, training and capacity building of the staff to reduce delays in initiating thrombolysis following admission is also important.

Our results show the necessity of optimizing STEMI care provided in peripheral hospitals in the 
management of acute STEMI with thrombolytic therapy as well as the need for creating awareness among the first contact care providers and the general public to achieve the recommended treatment times in more patients. ${ }^{11,12}$ Recognition of a possible myocardial infarction and the necessity to seek treatment immediately are keys to reducing delays in presentation and therefore total ischemic time.

In the absence of a STEMI registry or a systematized data collection in the setting, a convenient adhoc sampling was used. Hence the results are neither statistically significant nor they could be generalized for a wider population. However, this preliminary data from a peripheral hospital should help in designing more scientific audits, where the data can lead to more meaningful conclusions for STEMI care optimization.

\section{Conclusion}

Lessons learnt from this audit in a peripheral hospital indicate the need to optimize STEMI care at District General Hospitals. In the short term by establishing STEMI registries to generate more comprehensive and complete data and improving Door to Needle Time by introducing an institutional STEMI plan and appropriate staff training with post thrombolysis transfer protocols for rescue $\mathrm{PCI}$. Transfer protocols for a pharmaco-invasive strategy are also required. Creating awareness among the public and first contact care providers to reduce presenting delays and total Ischaemic Time also needs addressing. Introduction of newer thrombolytic agents that are fibrinspecific, that could be administered as a bolus and yield higher patency rates with establishment of comprehensive regional STEMI networks and care systems in parallel with Emergency Medical Services are necessary steps in the medium and long term to optimize STEMI care throughout Sri Lanka.

\section{References}

1. Gurugama P, Matthias AT, Rajkannah J, Kandeepan T, Indrakumar J, Gunatilake SB. Streptokinase in STEMI: ninety minute ECG for ST resolution; experience in an acute medical unit. Journal of the Ceylon College of Physicians 2012; 43 : 32-6.

2. Eric Boersma and the Primary Coronary Angioplasty vs. Thrombolysis (PCAT)-2 Trialists' Collaborative Group. Does time matter? A pooled analysis of randomized clinical trials comparing primary percutaneous coronary intervention and in-hospital fibrinolysis in acute myocardial infarction patients. European Heart Journal 2006; 27, 779-88.

3. Gersh BJ, Anderson JL. Thrombolysis and myocardial salvage: results of clinical trials and the animal paradigm paradoxic or predictable? Circulation 1993; 88: 296-306.

4. Gruppoltaliano per lo Studio Della Streptochinasinell' Infarto Miocardico (GISSI). Effectiveness of intravenous thrombolytic treatment in acute myocardial infarction. Lancet 1986; 1: 397-401.

5. ESC Guidelines for the management of AMI in patients presenting with ST-segment elevation. European Heart Journal 2012; 33, 2569-619.

6. 2013 ACCF/AHA Guideline for the Management of STElevation Myocardial Infarction. Circulation 2013; 127: e362-e425.

7. GiulioGuagliumi. Emerging data and decision for optimizing STEMI management: the European perspective. European Heart Journal Supplements 2009; 11(supplement C), C19-C24.

8. Alexander T, Mehta S, Mullasari A, Nallamothu BK. Systems of care for ST-elevation myocardial infarction in India. Heart 2012; 98: 15e17.

9. Otaall PS, Talwar KK. Limitations of currently available thrombolytic therapy. Indian Heart Journal 2009; 61: 47075 .

10. Chopra HK. The Place of tenecteplace in Management of STEMI. Indian Heart Journal 2009; 61: 408-9.

11. Soon CY, Chan WX, Tan HC. The impact of time-to-balloon on outcomes in patients undergoing modern primary angioplasty for acute myocardial infarction. Singapore Medical Journal 2007; 48(2): 131-6.

12. Burt DR. STEMI Boot Camp South Dakota Style: Apresentation made at "South Dakota Mission: Lifeline STEMI Summit" November 4, 2010. Accessed from http://www.heart.org/ HEARTORG on 22nd December 2014 